\title{
Energy-dependent collisional deactivation of vibrationally excited azulene
}

\author{
Jichun Shi and John R. Barkera) \\ Department of Atmospheric, Oceanic, and Space Sciences, Space Physics Research Laboratory, University \\ of Michigan, Ann Arbor, Michigan 48109-2143
}

(Received 4 January 1988; accepted 11 February 1988)

\begin{abstract}
Collisional energy transfer parameters for highly vibrationally excited azulene have been deduced from new infrared fluorescence (IRF) emission lifetime data with an improved calibration relating IRF intensity to vibrational energy [J. Shi, D. Bernfeld, and J. R. Barker, J. Chem. Phys. 88, 6211 (1988), preceding paper]. In addition, data from previous experiments [M. J. Rossi, J. R. Pladziewicz, and J. R. Barker, J. Chem. Phys. 78, 6695 (1983)] have been reanalyzed based on the improved calibration. Inversion of the IRF decay curves produced plots of energy decay, which were analyzed to determine $\langle\Delta E\rangle$, the average energy transferred per collision. Master equation simulations reproduced both the original IRF decays and the deduced energy decays. A third (simple) method of $\langle\Delta E\rangle$ determination agrees well with the other two. The results show $\langle\Delta E\rangle$ to be nearly directly proportional to the vibrational energy of the excited azulene from $\sim 8000$ to $33000 \mathrm{~cm}^{-1}$. At high energies, there are indications that the $\langle\Delta E\rangle$ energy dependence may be slightly reduced.
\end{abstract}

\section{INTRODUCTION}

Since the 1977 reviews by Tardy and Rabinovitch ${ }^{1}$ and by Quack and Troe, ${ }^{2}$ a higher order of knowledge has become available on polyatomic energy transfer. This new knowledge is the result of the development of physical methods (so-called "direct" methods) for energy transfer investigations involving large molecules in the electronic ground state. Up to about 1980, most energy transfer information for highly vibrationally excited molecules (HVEMs) was derived from unimolecular reaction studies near the low pressure limit. These studies were able to determine first order energy transfer properties that included the ordering of efficiencies of various collider gases and the magnitude of $\langle\Delta E\rangle$, the average energy transferred per collision. Some hints of higher order properties were also obtained, including indications that the stepladder model is more appropriate for efficient colliders and the exponential model is better for inefficient collision partners.

The new physical techniques do not rely on unimolecular reactions and they are therefore not affected by complicated reaction mechanisms and imperfect knowledge of the specific rate constants $[k(E)$ 's] for unimolecular reaction. Instead, they depend critically on accurate calibrations. The two physical methods used most widely are time-resolved infrared fluorescence (IRF) ${ }^{3}$ and ultraviolet absorbance (UVA), ${ }^{4}$ but time-dependent thermal lensing (TDTL), ${ }^{5-7}$ time-resolved optoacoustics (TROA), ${ }^{7,8}$ multiphoton ionization (MPI), ${ }^{9,10}$ stimulated emission pumping (SEP), ${ }^{11}$ atomic absorption line broadening, ${ }^{12}$ and other methods are now being developed and applied in various laboratories. Each method produces a time-resolved record of a physical property related to vibrational energy, or transferred energy; decay of the vibrational energy reflects the action of energy transfer, and the results can be expressed in terms of $\langle\Delta E\rangle$. Knowledge is more advanced for small molecules ${ }^{13}$ and elec-

\footnotetext{
"Address correspondence to this author.
}

tronically excited molecules, where propensity rules $^{14}$ have been identified.

The most detailed comparison between different physical techniques has involved the IRF and UVA methods. These techniques were both ${ }^{15,16}$ applied to azulene $\left(\mathrm{C}_{10} \mathrm{H}_{8}\right)$, which is an ideal molecule ${ }^{17}$ for photoexcitation studies because it can be excited by visible and ultraviolet light to the $S_{1}$ and $S_{2}$ electronic states, respectively, and each excited state undergoes rapid internal conversion to the $S_{0}$ ground state. Fluorescence and intersystem crossing are completely negligible for the $S_{1}$ state; intersystem crossing from the $S_{2}$ state is also negligible, while the quantum yield of fluorescence from the $S_{2}$ state is $\sim 3 \%$. Thus, azulene can be excited over a wide range of initial energies and it has very clean photophysics.

The IRF experiments using vibrationally excited azulene indicated that $\langle\Delta E\rangle$ depends approximately linearly on the vibrational energy content. ${ }^{15}$ This conclusion was reached after analyzing the initial IRF intensity decay in experiments carried out with 17000 and $30600 \mathrm{~cm}^{-1}$ initial energy, and by analyzing ${ }^{18}$ the intensity decay curve for some high-precision experiments at $\sim 30600 \mathrm{~cm}^{-1}$ initial energy. The interpretation of the experiments was based on a calibration curve for the energy-dependence of the infrared emission intensity from the azulene $\mathrm{C}-\mathrm{H}$ modes. The calibration curve was predicted by an elegant theory and was tested by experiments that showed agreement within experimental errors of about $\sim 30 \% .^{15,19}$

In contrast with the IRF results, the published UVA work ${ }^{16}$ on azulene indicates that $\langle\Delta E\rangle$ is only slightly dependent on vibrational energy. The UVA experiments were carried out at only one initial energy $\left(\sim 30600 \mathrm{~cm}^{-1}\right)$ and the interpretation was based on empirical calibration curves. Experiments in the same laboratory using other large molecules showed similar results, ${ }^{20}$ but other experiments with the UVA technique (on other molecules) have indicated that $\langle\Delta E\rangle$ is distinctly energy dependent. The discrepancy between the two physical techniques when applied to azu- 
lene is troubling and it shows that physical methods are not immune from problems, although the problems are different from those in unimolecular reaction studies.

To help identify the sources of the discrepancy, we carried out a complete experimental recalibration of the IRF dependence on vibrational energy, as described in paper $\mathbf{I}^{21}$ The experiments in paper I strongly confirm the validity of the IRF emission theory and the calculated theoretical calibration curve. In the course of that work, the calibration curve was better established than in previous work and new IRF decay data were obtained. The purpose of the present paper is analyze these new data to determine the energy transfer parameters. ${ }^{22}$ The older data ${ }^{15}$ are reconsidered and the derived energy transfer parameters are revised, based on the improved IRF calibration curve. As shown below, the revisions are relatively minor and the original conclusion is confirmed that $\langle\Delta E\rangle$ depends approximately linearly on vibrational energy. Recent UVA experiments on azulene ${ }^{23}$ (excited to lower energies than previously) also confirm a near-linear energy dependence up to $E \simeq 17000 \mathrm{~cm}^{-1}$.

\section{INFRARED FLUORESCENCE DECAY DATA}

The experimental details and results are fully described in paper $\mathrm{I}^{21}$ Azulene is excited with a pulsed tunable visibleultraviolet laser to the $S_{1}$ or $S_{2}$ electronically excited states. These states undergo fast internal conversion to produce the $S_{0}$ state with high vibrational excitation, which causes IRF emission to be produced. By varying the laser wavelength, the vibrational energy is varied, causing a variation in the initial IRF intensity. The dependence of the $\mathrm{C}-\mathrm{H}$ stretch mode IRF intensity on vibrational energy is the subject of paper I and is shown in Fig. 1.

Only about $2 \%$ of the azulene molecules in the laser beam are excited by the laser and these excited molecules predominantly undergo collisions with the unexcited azulene and are deactivated. If the amount of energy transferred per collision is less than $\sim 3000 \mathrm{~cm}^{-1}$, the $3000 \mathrm{~cm}^{-1}$ IRF

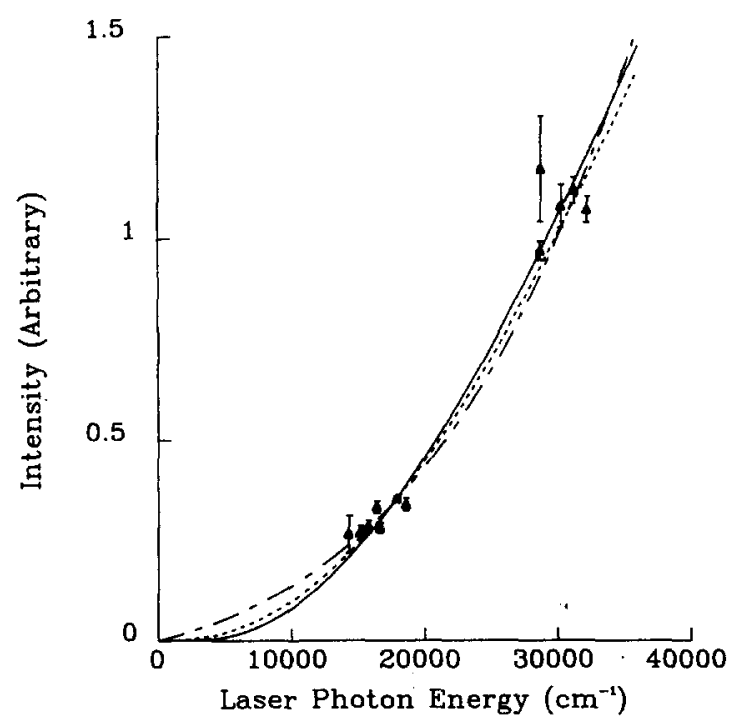

FIG. 1. IRF Calibration curve. Experimental points $( \pm \sigma)$ are shown; theoretical; --- Eq. (6); - Eq. (9).

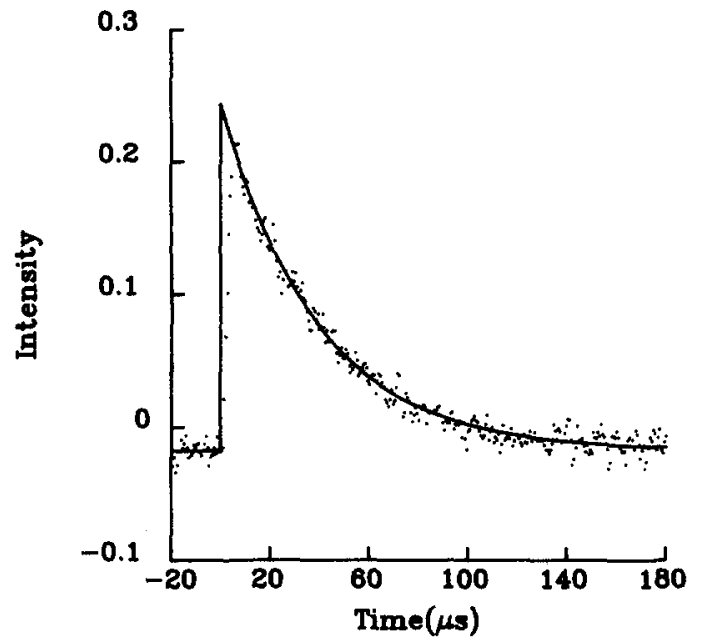

FIG. 2. IRF intensity decay. $6.2 \mathrm{mT}$ Torr azulene excited at $635 \mathrm{~nm}$.

from the initially excited molecule is decreased and that from the collision partner (which is now excited) is insignificant (see Fig. 1). Thus, the number of emitting molecules remains constant, but the IRF intensity decreases as the molecules are deactivated. This process explains the observed decay of the IRF intensity (Fig. 2), which can be used to deduce the magnitude and energy dependence of $\langle\Delta E\rangle$.

The observed IRF decays are found to be nearly exponential with time constant $\tau$. As shown earlier ${ }^{15}$ and in paper I, $\tau^{-1}$ is directly proportional to the concentration of collider and thus phenomenological bimolecular rate constants can be defined as $k=\left(\tau N_{\mathrm{Az}}\right)^{-1}$, where $N_{\mathrm{Az}}$ is the azulene number density. These rate constants depend on excitation energy and are summarized in Table $I$ and in Fig. 3. When other collider gases are added, as in earlier work with azulene, the rate constants depend on the pressure and nature of

TABLE I. Energy transfer analysis for pure azulene deactivation. Master equation simulation: $\langle\Delta E\rangle_{d}=295+0.034 \times E\left(\mathrm{~cm}^{-1}\right)$. Uncertainties are $\pm 1 \sigma$.

\begin{tabular}{cccc}
\hline \hline$h v\left(\mathrm{~cm}^{-1}\right)^{\mathrm{a}}$ & $10^{11} k\left(\mathrm{~cm}^{3} \mathrm{~s}^{-1}\right)$ & $\langle(E\rangle)\left(\mathrm{cm}^{-1}\right)^{\mathrm{b}}\langle\langle\Delta E\rangle\rangle\left(\mathrm{cm}^{-1}\right)^{\mathrm{b}}$ \\
\hline 14286 & $19.4 \pm 0.5$ & 12045 & $698 \pm 18$ \\
15152 & $18.3 \pm 0.7$ & 12736 & $709 \pm 26$ \\
15748 & $18.2 \pm 0.7$ & 13213 & $742 \pm 30$ \\
16393 & $18.6 \pm 0.7$ & 13725 & $797 \pm 31$ \\
16667 & $17.9 \pm 0.4$ & 13943 & $783 \pm 18$ \\
17094 & $17.2 \pm 0.8$ & 14281 & $779 \pm 35$ \\
17391 & $17.2 \pm 0.4$ & 14515 & $796 \pm 18$ \\
17896 & $17.8 \pm 0.8$ & 14912 & $854 \pm 37$ \\
18587 & $17.4 \pm 0.3$ & 15457 & $876 \pm 7$ \\
28730 & $14.2 \pm 0.1$ & 23304 & $1237 \pm 12$ \\
28776 & $13.3 \pm 0.7$ & 23339 & $1158 \pm 59$ \\
29674 & $13.3 \pm 0.9$ & 24023 & $1209 \pm 84$ \\
29940 & $13.7 \pm 0.7$ & 24226 & $1261 \pm 64$ \\
30303 & $13 \pm 0.5$ & 24502 & $1213 \pm 45$ \\
31250 & $13.9 \pm 0.3$ & 25220 & $1349 \pm 25$ \\
31746 & $13.7 \pm 0.1$ & 25598 & $1356 \pm 11$ \\
32258 & $13.2 \pm 0.4$ & 25982 & $1336 \pm 44$ \\
\hline \hline
\end{tabular}

a Laser excitation energy.

${ }^{\circ}-\langle\langle\Delta E\rangle\rangle=k\left[E_{0}-E(\tau)\right] / Z_{\mathrm{L}} ;\langle\langle E\rangle\rangle=\left[E_{0}+E(\tau)\right] / 2$. 


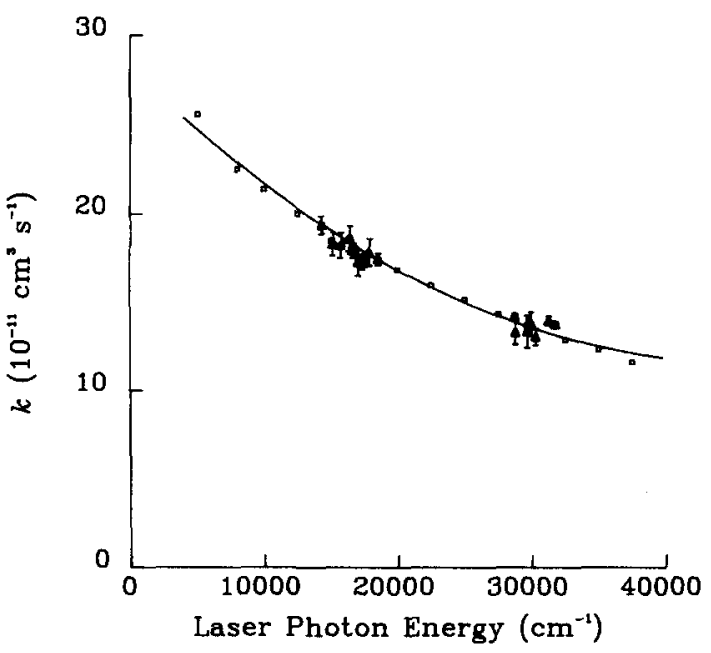

FIG. 3. $k$ vs laser photon energy. Solid points $( \pm \sigma)$ : experimental; open squares and solid line: Master equation simulations.

the collider gas; the rate constants obtained in the earlier work are also summarized in Table II.

For a detailed analysis, it is not desirable to assume an exponential decay. Although deviations from exponential behavior are small, ${ }^{15,18}$ it is better to use more flexible representations of the data, but this requires data sets of high precision. For this purpose, all of the decay curves (for the same pressure of azulene) obtained in the present work at a given excitation energy were weighted according to the number of laser shots and averaged to produce "super sets" of data with improved signal/noise. This procedure is equiva- lent to performing an experimental run with the number of laser shots equaling the sum of all the laser shots for the individual experiments.

\section{ENERGY TRANSFER ANALYSIS}

In this paper, three different methods are used to extract $\langle\Delta E\rangle$ values from the IRF data: Master equation simulations, "decay analysis", and "rate constant analysis". Each of these approaches is discussed and compared.

\section{Master equation simulations}

This approach uses a full Master equation treatment of the collisional problem to simulate the energy decay for laser excitation wavelength $\lambda$; the theoretical expression for $I(E)$ then predicts the IRF decay curve $I(\lambda, t)$, which is compared with the observed decay.

The Master equation implementation used here has been discussed in detail. ${ }^{24-26}$ Briefly, it uses exact-count ${ }^{27}$ densities of states based on a vibrational assignment for azulene, ${ }^{28}$ it obeys microscopic reversibility and detailed balance, and it can consider two assumed collision step-size distributions (exponential and reverse exponential). The initial population of excited molecules is in a Boltzmann distribution (at temperature $T$ ) that has been shifted to higher energy by absorption of a photon of energy $h v=c / \lambda$. Monte Carlo techniques are used to select initial conditions for the stochastic simulations.

In previous work, ${ }^{15}$ the azulene average thermal energy $\left(\sim 979 \mathrm{~cm}^{-1}\right.$ ) was added to the laser photon energy to obtain the excitation energy, Here, the thermal energy is correctly included by selecting the Boltzmann initial distribu-

TABLE II. Simple analysis" results for several collider gases excited at 600 and $337 \mathrm{~nm}$. Uncertainties are $\pm 1 \sigma$.

\begin{tabular}{lccccc}
\hline \hline Gas & $k(600)^{\mathrm{b}}$ & $k(337)^{\mathrm{b}}$ & $Z_{\mathrm{L}^{\mathrm{c}}}$ & $-\langle\langle\Delta E\rangle\rangle_{600}$ & $-\langle\langle\Delta E\rangle\rangle_{337}$ \\
\hline $\mathrm{Azu}$. & $17.89 \pm 0.41^{\mathrm{d}}$ & $13.6 \pm 0.3^{\mathrm{e}}$ & 12.4 & $783 \pm 18^{\mathrm{d}}$ & $1262 \pm 28^{\mathrm{e}}$ \\
$\mathrm{He}$ & $0.66 \pm 0.07$ & $0.69 \pm 0.09$ & 10.9 & $33 \pm 4$ & $73 \pm 10$ \\
$\mathrm{Ne}$ & $0.79 \pm 0.06$ & $0.72 \pm 0.12$ & 6.4 & $68 \pm 5$ & $130 \pm 22$ \\
$\mathrm{Ar}$ & $1.29 \pm 0.08$ & $1.07 \pm 0.03$ & 6.5 & $108 \pm 7$ & $190 \pm 5$ \\
$\mathrm{Kr}$ & $1.25 \pm 0.09$ & $0.86 \pm 0.04$ & 5.8 & $117 \pm 8$ & $171 \pm 8$ \\
$\mathrm{Xe}$ & $0.97 \pm 0.06$ & $0.92 \pm 0.06$ & 5.7 & $93 \pm 6$ & $187 \pm 12$ \\
$\mathrm{H}_{2}$ & $4.71 \pm 0.78$ & $3.14 \pm 0.16$ & 20.7 & $124 \pm 21$ & $175 \pm 9$ \\
$\mathrm{D}_{2}$ & $2.75 \pm 0.31$ & $1.74 \pm 0.09$ & 14.8 & $101 \pm 11$ & $136 \pm 7$ \\
$\mathrm{~N}_{2}$ & $1.48 \pm 0.11$ & $1.69 \pm 0.3$ & 7.5 & $108 \pm 8$ & $261 \pm 46$ \\
$\mathrm{O}_{2}$ & $1.56 \pm 0.1$ & $1.68 \pm 0.1$ & 7.2 & $119 \pm 8$ & $271 \pm 16$ \\
$\mathrm{CO}$ & $1.92 \pm 0.09$ & $2.2 \pm 0.1$ & 7.7 & $137 \pm 6$ & $332 \pm 15$ \\
$\mathrm{CO}$ & $3.54 \pm 0.57$ & $3.1 \pm 0.1$ & 7.7 & $250 \pm 40$ & $463 \pm 15$ \\
$\mathrm{H}_{2} \mathrm{O}$ & $5.6 \pm 0.59$ & $6.92 \pm 0.85$ & 12.3 & $247 \pm 26$ & $647 \pm 79$ \\
$\mathrm{NH}_{3}$ & $5.09 \pm 0.46$ & $7.59 \pm 0.52$ & 12 & $231 \pm 21$ & $729 \pm 50$ \\
$\mathrm{CH}_{4}$ & $3.1 \pm 0.13$ & $3.7 \pm 0.36$ & 10.8 & $157 \pm 7$ & $397 \pm 39$ \\
$n-\mathrm{C}_{4} \mathrm{H}_{10}$ & $11.7 \pm 1.2$ & $9.41 \pm 0.28$ & 9.9 & $641 \pm 66$ & $1093 \pm 33$ \\
$\mathrm{SF}_{6}$ & $3.9 \pm 0.3$ & $3.14 \pm 0.1$ & 6.7 & $319 \pm 25$ & $543 \pm 17$ \\
\hline \hline
\end{tabular}

' $\langle\langle\Delta E(\langle\langle E\rangle\rangle)\rangle\rangle=-k\left[E_{0}-E(\tau)\right] / Z_{\mathrm{L}}$, where $\left[E_{0}-E(\tau)\right]=11541$ and $5448 \mathrm{~cm}^{-1}$ at 337 and 600 nm, respectively; $\langle\langle E\rangle\rangle=\left[E_{0}+E(\tau)\right] / 2$, where $\langle\langle E\rangle\rangle_{600}$ and $\langle\langle E\rangle\rangle_{337}$ are 13943 and $24023 \mathrm{~cm}^{-1}$, respectively.

${ }^{b}$ From Ref. 15, unless otherwise noted; units: $10^{-11} \mathrm{~cm}^{3} \mathrm{~s}^{-1}$

${ }^{c}$ Lennard-Jones collision frequencies ${ }^{15}$; units: $10^{-10} \mathrm{~cm}^{3} \mathrm{~s}^{-1}$.

${ }^{\mathrm{d}}$ This work and paper $I$.

'Compare with new data in Table 1 . 
tion. In the figures, "excitation energy" means either laser photon energy or microcanonical energy, depending on the context; the difference between these two quantities is small and so we will use the terms interchangeably.

Energy transfer is described in terms of $\langle\Delta E\rangle_{d}$, the average stepsize for deactivating collisions, which is assumed to have the following energy dependence:

$$
\langle\Delta E\rangle_{d}=\alpha+\beta E,
$$

where $\alpha$ and $\beta$ are parameters. More flexible expressions for $\langle\Delta E\rangle_{d}$ could be used, if required. The relationships between $\langle\Delta E\rangle$ and $\langle\Delta E\rangle_{d}$ for the exponential and reverse-exponential models have been discussed elsewhere. ${ }^{24,25}$

For photoexcitation at wavelength $\lambda$, the energy at each step of a stochastic trajectory is used with $I(E)$ to predict $I(\lambda, t)$ for the individual trajectory, and the results from many trajectories are combined to produce the predicted IRF decay curve corresponding to the laser excitation energy and selected values for $\alpha$ and $\beta$. The predicted IRF decay curves are fitted to exponentials by the same nonlinear least squares method as used for the experimental data, ${ }^{21}$ and the simulated and experimental phenomenological bimolecular rate constants are compared. If the comparison is not satisfactory simultaneously for simulations at two laser excitation wavelengths ( 635 and $322.3 \mathrm{~nm}$ ), new energy transfer parameters are selected and the trial is repeated until satisfactory agreement is obtained.

The results of the Master equation can be presented in several ways, which include the predicted IRF decay, the average energy decay $\langle E(t)\rangle,\langle\Delta E(\langle E\rangle)\rangle_{e}$ derived from the energy decay, and $\langle\Delta E(E)\rangle_{m}$ for a microcanonical ensemble. The average energy decay can be written ${ }^{15}$

$$
\frac{d\langle E\rangle}{d t}=Z_{\mathrm{LJ}} N_{\mathrm{Az}}\langle\Delta E(t)\rangle_{e}
$$

where $\langle\Delta E(t)\rangle_{e}$ can be expressed as $\langle\Delta E(\langle E\rangle)\rangle_{e}$, because $\langle E(t)\rangle$ is determined in the same Master equation simulations.

The excitation energies and experimental rate constants are presented in Table I along with the $\langle\Delta E\rangle_{d}$ expression that best simulated the data. When this expression is used for excitation energies between 10000 and $40000 \mathrm{~cm}^{-1}$, the agreement between the observed and simulated rate constants is excellent for the entire range of laser photon energies, as shown in Fig. 3. The microcanonical $\langle\Delta E(E)\rangle_{m}$ and the ensemble average $\langle\Delta E(\langle E\rangle)\rangle_{e}$ from computer simulations are presented in Fig. 4 for comparison and it is seen that both methods indicate that $\langle\Delta E\rangle$ depends approximately linearly on vibrational energy.

\section{Decay analysis}

The IRF decay curves can be analyzed directly in several ways. One method is to use the calibration curve relating IRF intensity to vibrational energy and convert each IRF intensity datum to the corresponding vibrational energy, producing a plot of vibrational energy vs time. ${ }^{18}$ To carry out this procedure, the IRF decay curves were least squares fitted to a fifth order polynomial, neglecting data earlier than 5 $\mu$ s, because the signals are limited by the detector rise time. ${ }^{21}$ The polynomial fit is used to determine the initial IRF inten-

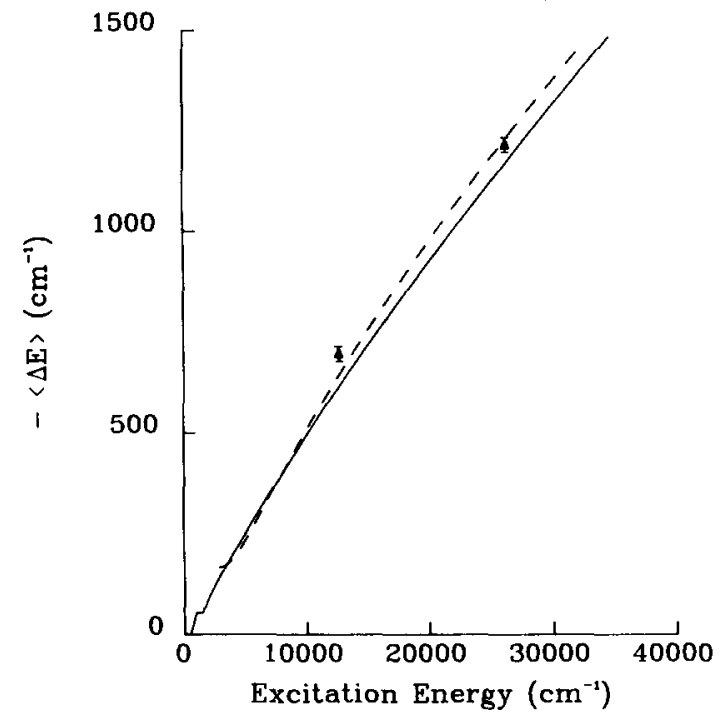

FIG. 4. Master equation simulations. - Microcanonical $\langle\Delta E\rangle_{;}--\langle\Delta E\rangle$ from energy decay; solid points: $\langle\Delta E\rangle$ from simple analysis.

sity at $t=0$, and this initial intensity is then scaled to the $I(E)$ calibration curve at the known laser excitation energy.

The calibration curve used in this procedure was that for the $300 \mathrm{~K}$ thermal average. ${ }^{21}$

$$
\begin{aligned}
& \langle I(h v, T)\rangle \\
& \quad=\frac{1}{Q(T)} \int_{0}^{\infty} I(E+h v) \rho_{s}(E) \exp \left(-\frac{E}{k T}\right) d E,
\end{aligned}
$$

where $I(E)$ is the theoretical calibration curve [Eq. (8), paper I,$Q(T)$ is the vibrational partition function, $\rho_{s}(E)$ is the density of states for all $s=48$ vibrational modes, and $h v$ is the laser photon energy. This averaged function was used because the thermal distribution superficially resembles the ensemble distribution of excited azulene molecules as they are deactivated. Analysis of several calibration curves derived by different methods showed that the differences among them are small. ${ }^{18}$ Moreover, Eq. (3) is a smoother function than the microcanonical expression, simplifying the conversion from IRF intensity to energy.

Two examples of this decay analysis procedure are shown in Fig. 5. The results show that the energy decays

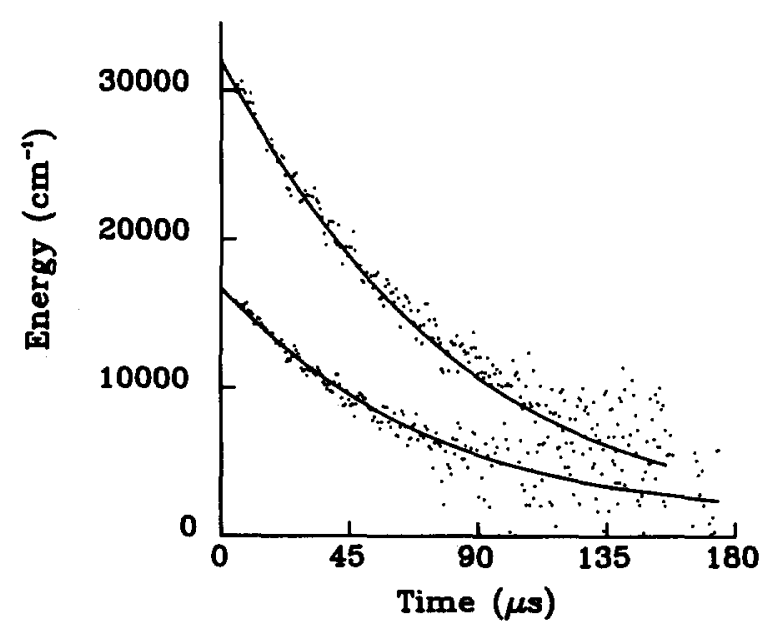

FIG. 5. Energy decays for 635 and $322.5 \mathrm{~nm}$ excitation. Points: from converting intensity to energy; lines: Master equation simulations. 


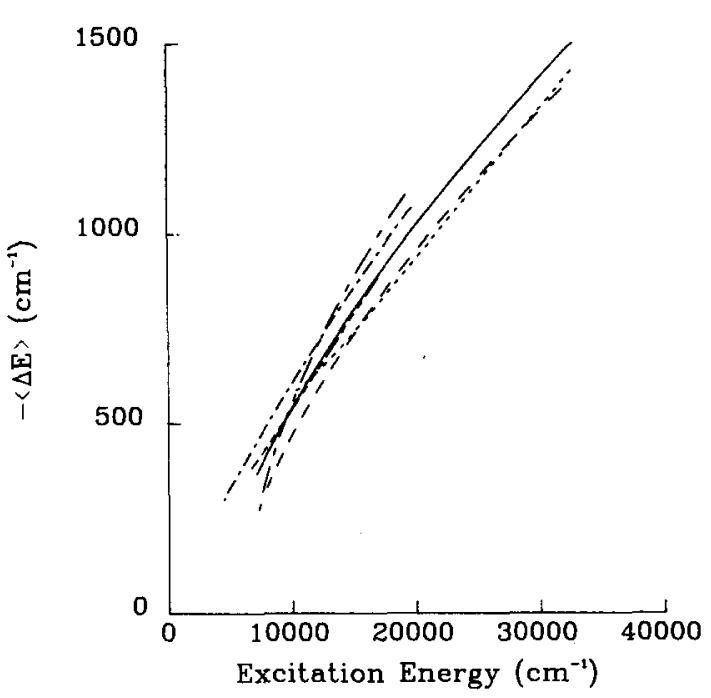

FIG. $6 .\langle\Delta E\rangle$ from decay analysis. $315 \mathrm{~nm}-; 310 \mathrm{~nm}$---; $322.3 \mathrm{~nm} \mathrm{-} \mathrm{-} \mathrm{-} \mathrm{;}$ $558 \mathrm{~nm}-\cdots-; 538 \mathrm{~nm}---; 635 \mathrm{~nm}-\cdots$.

nearly exponentially, as deduced previously from other IRF data. ${ }^{15,18}$ The solid lines are the results of Master equation simulations of two of the experimental runs, showing the excellent agreement between the simulations and the experimental energy decays even at low energies, where noise interference is greatest. The energy decay curves were fitted with third-order polynomials and differentiated to obtain $\langle\Delta E(t)\rangle_{e}$ and corresponding $\langle\Delta E(E)\rangle_{e}$ from Eq. (2). The $\langle\Delta E(E)\rangle$ values deduced by this procedure from the simulated IRF decay data produced with the Master equation calculations is shown in Fig. 4 for comparison with the $\langle\Delta E\rangle$ values from the various methods: the results show excellent agreement and self-consistency.

Experimental runs treated in the same way are presented in Fig. 6. The experimental lines show very good agreement and the results indicate that $\langle\Delta E\rangle$ is approximately proportional to the vibrational energy content. It is particularly noteworthy that the results from lower-energy excitations are completely consistent with those from higher energies.

\section{Rate constant analysis}

The simplest (but most approximate) way to extract $\langle\Delta E\rangle$ values from the data is to use the phenomenological bimolecular rate constants, Lennard-Jones collision frequencies $Z_{\mathrm{LJ}}$, and the IRF calibration curve. Peviously, it was shown that $\langle\Delta E\rangle$ at the initial energy can be approximated by the expression ${ }^{15}$

$$
\langle\Delta E\rangle=-\frac{k}{Z_{\mathrm{L}}}\left(I \frac{d E}{d I}\right)_{0},
$$

where the factor in brackets is obtained from the IRF calibration curve. This expression is only moderately accurate, because it depends on the derivative, which varies strongly with energy. When the IRF intensity decays to $1 / e$ of the initial value, the corresponding energy change produces a substantial variation in the derivative. We have found that a

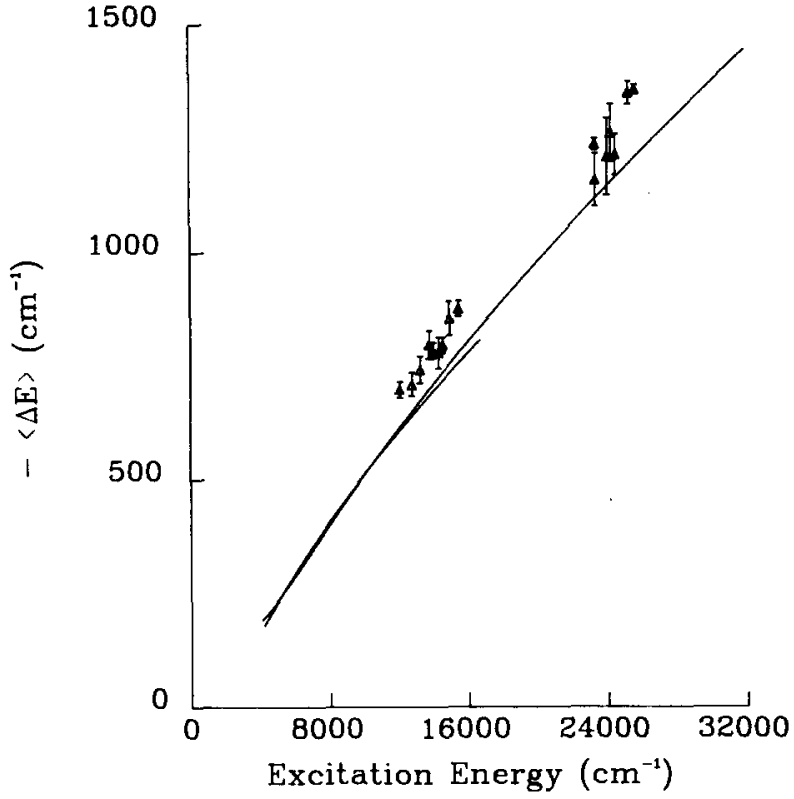

FIG. 7. Simple analysis $\langle\Delta E\rangle$ vs photon energy. Points $( \pm \sigma)$ : experimental data; solid lines: Master equation simulations for excitation at 636 and $322.3 \mathrm{~nm}$.

more accurate expression considers the energy decay during the time corresponding to the $1 / e$ intensity decay

$$
\langle\langle\Delta E(\langle\langle E\rangle\rangle)\rangle\rangle=-\frac{k}{Z_{\mathrm{LJ}}}\left[E_{0}-E_{\tau}\right] .
$$

Here, the double bracket emphasizes the averaging involved in this simple analysis; $Z_{\mathrm{LJ}}$ is the Lennard-Jones bimolecular rate constant, $E_{0}$ is the initial excitation energy ( $I_{0}$ is the corresponding initial intensity), $E_{\tau}$ is the energy corresponding to $I(\tau)=I_{0} / e$, according to the IRF calibration curve, and $\langle\langle E\rangle\rangle=\left(E_{0}+E_{\tau}\right) / 2$.

The $\langle\Delta E\rangle$ values calculated from this simple analysis applied to the Master equation simulations agree very well with the values obtained by the other two methods, as shown in Fig. 4. (Even better agreement might be obtained using the simple analysis if an exponential energy decay is assumed, rather than linear decay implicitly assumed in the definition of $\langle\langle E\rangle\rangle$.) The numerical values for the terms in Eq. (5) are summarized in Table I and the resulting $\langle\langle\Delta E\rangle\rangle$ values are presented in the table and in Fig. 7. All of these results show $\langle\Delta E\rangle$ to be approximately proportional to vibrational energy. The rate constant data reported earlier ${ }^{15}$ were also analyzed using this approach and the results again show $\langle\Delta E\rangle$ to be approximately proportional to vibrational energy (Table II).

Note that $\langle\Delta E\rangle$ is derived from the measurements on the basis of assumed Lennard-Jones collision frequencies and its magnitude therefore depends on the assumed $Z_{\mathrm{LJ}}$; for comparison, the values for $Z_{L J}$ in the present work and in Ref. 15 are slightly larger than those in Ref. 16.

\section{Sensitivity to fitting function}

All of the analyses carried out above are based on the theoretical calibration curve. In particular, the shape of the calibration curve is important in the $\langle\Delta E\rangle$ analysis. Al- 
though it was shown in paper I that the experimental results are in excellent agreement with the theory describing infrared emission, it is useful to examine empirical calibration curves that are consistent with the experimental data, but may significantly affect the deduced $\langle\Delta E\rangle$ energy dependence. $^{16}$

Several conditions must be fulfilled by empirical calibration functions. In the experiments, the observables are laser photon energy and the difference between the IRF from the excited molecules and the normal thermal azulene. Thus, the function must vanish for laser photon energy equal to zero (note that a simple exponential ${ }^{16}$ does not satisfy this condition). The calibration data were fitted with four empirical functions that vanish for laser photons with zero energy

$$
\begin{aligned}
& I=A(h v)^{n}, \\
& I=A(h v)^{n(E),} \\
& I=A(1+a h v)(h v)^{n}, \\
& I=A[\exp (-b h v)-1] .
\end{aligned}
$$

The calibration curves obtained with Eqs. (6) and (9), and the theoretical calibration are shown in Fig. 1 for comparison. Empirical equation (6) qualitatively resembles the theoretical curve, except at low photon energy. Equation (9) shows strong deviations from the theoretical curve at all intermediate energies. In particular, Eq. (9) predicts substantial IRF at very low photon energies, even though the azulene thermal vibrational energy is only about $1000 \mathrm{~cm}^{-1}$. This prediction is nonphysical because below a laser photon energy of $\sim 2000 \mathrm{~cm}^{-1}$, most molecules cannot emit at 3000 $\mathrm{cm}^{-1}$. Despite this failure, Eq. (9) still fits the experimental data very well.

These calculations show that the experimental calibration data cannot be used alone to clearly distinguish between different empirical functions. Because azulene can only be excited at wavelengths where it absorbs, the calibration data

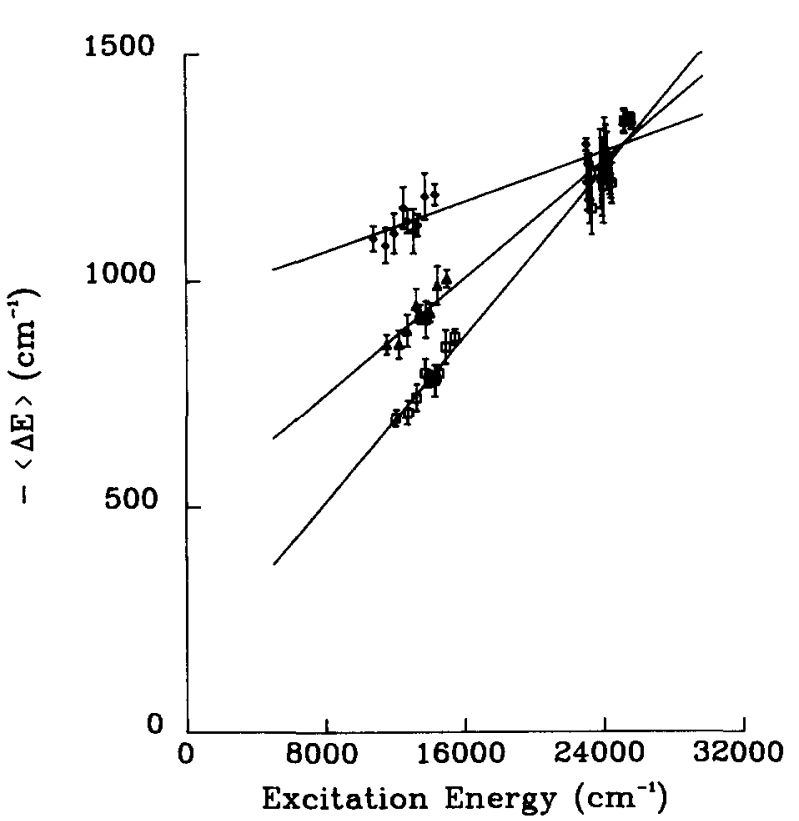

FIG. 8. Effect of calibration curve shape. From top to bottom: analysis according to Eq. (9), Eq. (6), and theory. are obtained in only two limited energy ranges where the absorption coefficient is large and the photophysics uncomplicated. This fact, coupled with the experimental uncertainties, eliminates the possibility of finding a unique empirical calibration, even though the error bars are relatively small. Thus we are fortunate that the theory for IRF from vibrationally excited polyatomics is both well developed and tractable.

Values for $\langle\Delta E\rangle$ were calculated from each of the empirical calibration curves by using the simple analysis. The results using Eqs. (6) to (8) were quantitatively quite similar and showed slightly larger $\langle\Delta E\rangle$ values than the theoretical calibration at low vibrational energies, as shown in Fig. 8 for Eq. (6). In contrast, the $\langle\Delta E\rangle$ values obtained using Eq. (9) show very little dependence on vibrational energy; these values are very unlikely to be correct, however, because the empirical calibration function is nonphysical. Nonetheless, it is clear that the conclusions regarding $\langle\Delta E\rangle$ depend strongly on choice of calibration curve.

Since the theoretical calibration curve is based firmly on well-established principles and because it is completely consistent with the experimental data, ${ }^{21}$ it is greatly preferred over the empirical functions.

\section{Reanalysis of previous $\langle\Delta E\rangle$ data}

In the present work, the theoretical calibration curve is somewhat different from that used previously, ${ }^{15}$ which was based on the Whitten-Rabinovitch approximation ${ }^{29}$ for densities of states. The differences are small, but we have reanalyzed the experimental data obtained earlier ${ }^{15}$ to arrive at revised values for $\langle\Delta E\rangle$, as summarized in Table II. In earlier work, ${ }^{15}$ azulene pressure measurements were not reliable, leading to an error in $\langle\Delta E\rangle$ for deactivation by azulene measured at $600 \mathrm{~nm}$, as discussed previously. ${ }^{18}$ This error did not affect $\langle\Delta E\rangle$ values for other collider gases, because

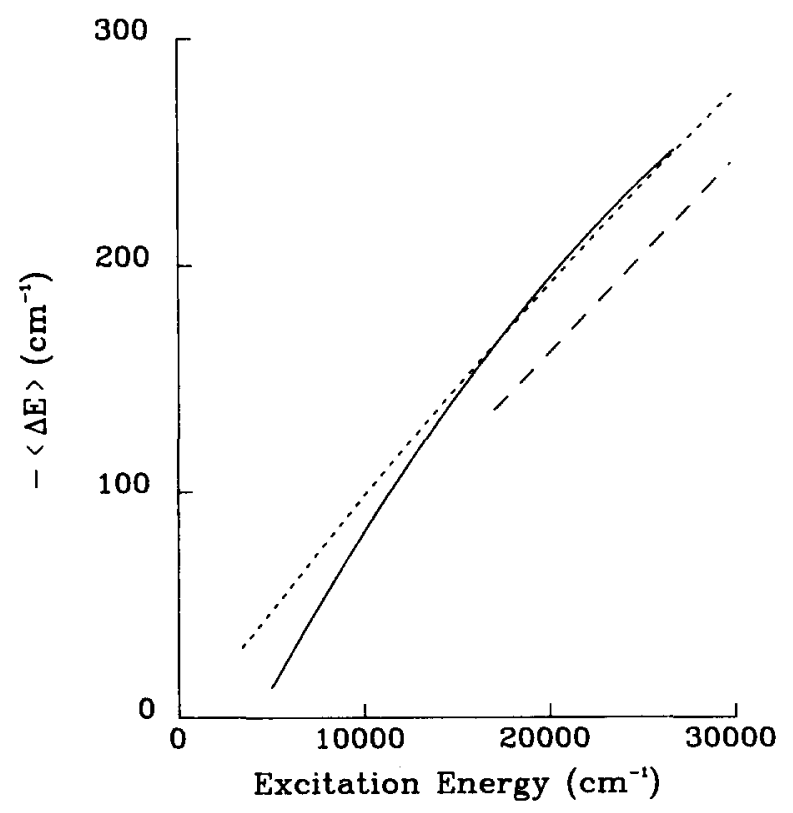

FIG. 9. $\langle\Delta E\rangle$ for $8.9 \mathrm{mTorr}$ azulene in $288 \mathrm{mTorr}$ argon at $337 \mathrm{~nm}$. Decay analysis of experiment; --- Master equation simulation; - - Forst and Barker. $^{18}$ 
the pressures were higher and the measurement problems were not as severe.

Previously, several experiments were run for exceptionally large numbers of laser shots to obtain high-precision data. ${ }^{15}$ These data were analyzed ${ }^{18}$ using the decay analysis method and it was concluded that deactivation by argon gives a $\langle\Delta E\rangle$ that depends linearly on vibrational energy. An analysis of the same data set with the theoretical calibration curve is shown in Fig. 9 along with a Master equation simulation ( $\alpha=100, \beta=0.009$ ). Also shown for comparison is the result obtained from an earlier analysis ${ }^{18}$ that used the old version of the theoretical calibration. The results show good quantitative agreement.

\section{DISCUSSION}

\section{Energy-dependent $\langle\Delta E\rangle$}

The present work confirms the earlier conclusion that, for azulene, $\langle\Delta E\rangle$ is a nearly linear function of vibrational energy. All three methods of analysis are self-consistent and even the simple analysis method gives good results. The simple analysis results for deactivation of azulene by several collider gases are presented in the tables and each collider gas exhibits an energy-dependent $\langle\Delta E\rangle$. The more detailed decay analysis results for azulene self-deactivation and for deactivation by argon clearly show the near-linear energy dependence. In each case, the decay curve is slightly concave, which reduces the energy dependence at high energy. Over the range of these experiments $\left(5000 \mathrm{~cm}^{-1} \leqslant E \leqslant 33000\right.$ $\mathrm{cm}^{-1}$ ) the change in slope is small and uncertain, considering the experimental uncertainties.

A full Master equation simulation with energy-dependent $\langle\Delta E\rangle_{d}$ satisfactorily reproduces the IRF emission decay, the inferred energy decay, and the energy dependence of $\langle\Delta E\rangle$. Also, the simulated $\langle\Delta E\rangle$ function qualitatively shows the same curvature exhibited by the experimental data, indicating that $\langle\Delta E\rangle_{d}$ (as distinguished from $\langle\Delta E\rangle$ ) may be a linear function of energy and the curvature in the $\langle\Delta E\rangle$ vs $E$ plot is a consequence of detailed balance. The fact that the curvature is seen consistently in both the high energy and low energy excitation experiments and in the Master equation simulations tends to indicate that it is really present, but small enough to be neglected over this energy range.

\section{Comparisons with other work}

All of the analyses using the theoretical calibration curve show that $\langle\Delta E\rangle$ for excited azulene deactivation by unexcited azulene is approximately proportional to vibrational energy. Although simple models of energy transfer qualitatively predict energy dependence, ${ }^{30}$ the predictions are suspect, because the predicted magnitudes of $\langle\Delta E\rangle$ are factors of 3 to 10 too large. The biased random walk theory ${ }^{31}$ is much more sophisticated and it predicts an energy-dependent $(\Delta E)$ for deactivation of azulene by argon that is close to the observed magnitude, but is slightly less dependent on vibrational energy. Thus, the energy dependence of $\langle\Delta E\rangle$ is not surprising from a theoretical point of view.

Experiments using molecules other than azulene have produced a wide range of results. For example, UVA experi- ments on cycloheptatriene derivatives and toluene indicate that $\langle\Delta E\rangle$ is nearly independent of energy, ${ }^{20}$ but UVA experiments on triatomics ${ }^{32}$ and on benzene derivatives ${ }^{33,34}$ indicate that $\langle\Delta E\rangle$ is approximately proportional to internal energy. Optoacoustic ${ }^{8}$ and line-broadening ${ }^{12}$ experiments on several molecules show that $\langle\Delta E\rangle$ is an approximately linear function of energy. Thus, different conclusions are reached when different molecules are investigated and no general rule is apparent. Clearly, considerable progress is needed on both experiments and theory, before polyatomic energy transfer will be understood.

The present work confirms the previous results obtained with the IRF method, but all of this work contrasts with published UVA results for azulene, ${ }^{16}$ which show little energy dependence, except at low vibrational energy. These UVA experiments were performed with excitation only at $337 \mathrm{~nm}$ and the interpretation depends on a decay analysis and on an empirical calibration curve. Recently, new UVA results were obtained near $600 \mathrm{~nm}$ excitation and the decay analysis (based on the same empirical calibration curve) indicates that $\langle\Delta E\rangle$ is approximately proportional to vibrational energy, ${ }^{23}$ in contrast with the higher excitation energy UVA experiments. The discrepancy with the earlier UVA work is minimized if $\langle\Delta E\rangle$ is nearly independent of vibrational energy between 17000 and $30000 \mathrm{~cm}^{-1}$, but proportional to vibrational energy at lower energies: i.e., there is a "saturation" effect. The IRF decay analysis in the present work for both azulene and argon collider gases shows a very small decrease in slope of the $\langle\Delta E\rangle$ vs energy curve as the energy is increased and there is no sudden saturation behavior in the range of the present experiments.

In other unpublished results obtained using azulene, multiphoton excitation has been used to excited azulene to $\sim 60000 \mathrm{~cm}^{-1}$ and the extent of its isomerization to napthalene has been measured for various collider gas pressures. ${ }^{35}$ The energy transfer analysis depends on the specific rate constants $[k(E)$ 's] for isomerization, which were inferred from shock tube measurements of the thermal isomerization rate constant. ${ }^{36}$ It was concluded that $\langle\Delta E\rangle$ has about the same magnitude at $60000 \mathrm{~cm}^{-1}$ as at $30000 \mathrm{~cm}^{-1}$, and thus that $\langle\Delta E\rangle$ is constant for energies greater than $30000 \mathrm{~cm}^{-1}$. This conclusion is highly uncertain, however, because the $k(E)$ 's were only determined to within a factor of $2^{36}$ and the change in $\langle\Delta E\rangle$ is predicted by the present work to be less than that. Furthermore, the $k(E)$ 's were obtained from the shock tube data only after falloff corrections that required assumed values for collision efficiency, which depends on $\langle\Delta E\rangle$. Thus, the analysis of $\langle\Delta E\rangle$ in the multiphoton experiments relies on $k(E)$ 's are uncertain and were obtained only after making prior assumptions about $\langle\Delta E\rangle$. The multiphoton excitation results are preliminary and inconclusive, but the technique shows promise, if it is free of complications.

Other experiments on azulene energy transfer do not directly address the presence, or absence of $\langle\Delta E\rangle$ energy dependence: a time-resolved optoacoustic experiment on azulene $^{37}$ showed that $V-T$ energy transfer occurs on about the same time scale as the deactivation, in agreement with a time-dependent thermal lensing study ${ }^{6}$ of the molecule.

In summary, both the IRF results and the recent UVA 
experiments on azulene at low excitation energy support the conclusion that $(\Delta E)$ is approximately a linear function of vibrational energy. However, the UVA results at higher energy do not agree and the origin of this discrepancy has not been identified, although it may be connected with the empirical calibration curves used in the UVA work. A recalibration of the UVA experiments would seem to be in order.

\section{Consequences of energy-dependent $\langle\Delta E\rangle$}

The collisional decay of internal energy is described by $\langle\Delta E\rangle$. When $\langle\Delta E\rangle$ is a linear function of energy in a nonreactive system, the ensemble average energy $\langle E\rangle$ decays exponentially, if the initial distribution is a delta function. ${ }^{18,38}$ The present photoexcitation experiments produce a near-delta function of initial energy and the energy-decay curves show near-exponential decay (Fig. 5). The near-exponential energy decay is reflected in the near-linear energy dependence of the derived $\langle\Delta E\rangle$ values.

In investigations of the temperature dependence of $\langle\Delta E\rangle$, recognition of the effect of the vibrational energy dependence is very important. As the temperature is raised, the average vibrational energy content increases and photoexcitation will produce more highly excited molecules than at low temperature. The effect of raising the vibrational energy must be disentangled from potential thermal effects that result from higher temperature collider gas and changed rotational energy distributions. This is the reason simple comparisons among different experiments are not always possible and why Master equation simulations were used in the IRF investigation of temperature effects. ${ }^{25}$

Another important consequence of the energy-dependent $\langle\Delta E\rangle$ is that energy transfer is more effective at high than at low internal energy. Pritchard has commented ${ }^{39}$ that there is a large discrepancy between the vibrational relaxation times observed in shock tube experiments and the assumed collisional deactivation rates assumed by RRKM theory, indicating a failure of RRKM theory. This discrepancy has motivated him to develop alternatives to RRKM theory that invokes collisional "randomization" of energy, instead of the conventional collisionless intramolecular energy redistribution.

It is possible, however, that an energy-dependent $\langle\Delta E\rangle$ can account for the apparent discrepancy. The vibrational relaxation time (related to the "incubation time"40) is the time needed for the internal vibrational energy of the reactive molecules to readjust to the sudden collider bath temperature change induced by the shock wave. If the initial energy is very low, as in most such experiments, the molecule must be activated by a long sequence of collisions to energies above the reaction threshold. At low energies, the activation process will be very inefficient and slow, because of the small magnitude of $\langle\Delta E\rangle$ and the vibrational relaxation time will be long. However, collisional deactivation of excited molecules from energies just above to just below the reaction threshold (and vice versa) will be efficient and rapid, because the energy-dependent $\langle\Delta E\rangle$ has a large magnitude at these energies. Master equation calculations to address this question quantitatively are now underway in this laboratory.

\section{ACKNOWLEDGMENTS}

The authors thank the Department of Energy (Office of Basic Energy Sciences) for funding. Thanks also go to R. J. Gordon, J. Troe, H. Hippler, and R. W. Carr for preprints.

'D. C. Tardy and B. S. Rabinovitch, Chem. Rev. 77, 369 (1977)

${ }^{2} \mathrm{M}$. Quack and J. Troe, Specialists Periodical Reports 2, 175 (1977)

${ }^{3}$ G. P. Smith and J. R. Barker, Chem. Phys. Lett. 78, 253 (1981).

${ }^{4}$ H. Hippler, J. Troe, and H. J. Wendelken, Chem. Phys. Lett. 84, 257 (1981).

${ }^{5}$ F. R. Grabiner, D. R. Siebert, and G. W. Flynn, Chem. Phys. Lett. 17, 189 (1972); D. R. Siebert, F. R. Grabiner, and G. W. Flynn, J. Chem. Phys. 60, 1564 (1974).

${ }^{6}$ J. R. Barker and T. Rothem, Chem. Phys. 68, 331 (1982); P. L. Trevor, T. Rothem, and J. R. Barker, ibid. 68, 341 (1982).

${ }^{7}$ J. R. Barker and B. M. Toselli, in Photothermal Investigations of Solids and Fluids, edited by Jeffrey A. Sell (Academic, New York, in press).

${ }^{8}$ N. Presser, J. R. Barker, and R. J. Gordon, J. Chem. Phys. 78, 2163 (1983); K. M. Beck, A. Ringwelski, and R. J. Gordon, Chem. Phys. Lett. 121, 529 (1985).

${ }^{9}$ K. Luther, K. Reihs, and J. C. Rodriguez-Morillo, Collisional Energy Transfer Studied by Multiphoton Ionization, 10th International Conference on Molecular Energy Transfer, Emmetten, Switzerland, 23-28 August, 1987.

${ }^{10}$ J. E. Sabol and R. W. Carr (preprint, 1987).

"F. Temps, F. Halle, P. H. Vaccaro, J. L. Kinsey, and R. W. Field, Collisional Energy Transfer in Highly Vibrationally Excited $\mathrm{H}_{2} \mathrm{CO}\left(X^{1} A_{1}\right)$ ", 10th International Conference on Molecular Energy Transfer, Emmetten, Switzerland, 23-28 August, 1987.

${ }^{12}$ T. J. Wallington, M. D. Scheer, and W. Braun, Chem. Phys. Lett. 138, 538 (1987).

${ }^{13}$ J. T. Yardley, Introduction to Small Molecule Energy Transfer (Academic, New York, 1980), p. 292.

${ }^{14}$ For a recent review, see D. J. Krajnovich, C. S. Parmenter, and D. L. Catlett, Jr., Chem. Rev. 87, 237 (1987).

${ }^{15}$ M. J. Rossi, J. R. Pladziewicz, and J. R. Barker, J. Chem. Phys. 78, 6695 (1983), and references therein.

${ }^{16}$ H. Hippler, L. Lindemann, and J. Troe, J. Chem. Phys. 83, 3906 (1985).

${ }^{17}$ For azulene photophysics references, see paper I.

${ }^{18}$ W. Forst and J. R. Barker, J. Chem. Phys. 83, 124 (1985).

${ }^{19} \mathrm{M}$. J. Rossi and J. R. Barker, Chem. Phys. Lett. 85, 21 (1982).

${ }^{20} \mathrm{H}$. Hippler, J. Troe, and H. J. Wendelken, J. Chem. Phys. 78, 5351, 6709, 6718 (1983), M. Heyman, H. Hippler, and J. Troe, ibid. 80, 1853 (1984).

${ }^{21}$ Preceeding paper, called paper I: J. Shi, D. Bernfeld, and J. R. Barker, J. Chem. Phys. 88, 6211 (1988).

${ }^{22}$ A preliminary report of these results appeared in J. R. Barker, Deactivation of Large Molecules in the Electronic Ground State, 10th International Conference on Molecular Energy Transfer, Emmetten, Switzerland, 23-28 August, 1987.

${ }^{23} \mathrm{~J}$. Troe, Collisional Energy Transfer in Chemical Reactions, 10th International Conference on Molecular Energy Transfer, Emmetten, Switzerland, 23-28 August, 1987; J. Troe and H. Hippler (private communication).

${ }^{24}$ J. R. Barker, Chem. Phys. 77, 301 (1983).

${ }^{25}$ J. R. Barker and R. E. Golden, J. Phys. Chem. 88, 1012 (1984).

${ }^{26}$ J.-M. Zellweger, T. C. Brown, and J. R, Barker, J. Chem. Phys. 83, 6251 (1985).

${ }^{27}$ S. E. Stein and B. S. Rabinovitch, J. Chem. Phys. 58, 2438 (1973).

${ }^{28}$ R. S. Chao and R. K. Kahnna, Spectrochim. Acta Part A 33, 53 (1977).

${ }^{29}$ G. Z. Whitten and B. S. Rabinovitch, J. Chem. Phys. 41, 1883 (1964).

${ }^{30}$ See Ref. 15 for a discussion.

${ }^{31}$ R. G. Gilbert, J. Chem. Phys. 80, 5501 (1984); K. F. Lim and R. G. Gilbert, ibid. 84, 6129 (1986).

${ }^{32}$ J. E. Dove, H. Hippler, and J. Troe, J. Chem. Phys. 82, 1907 (1985)

${ }^{33} \mathrm{~N}$. Nakashima and K. Yoshihara, J. Chem. Phys. 77, 6040 (1982); ibid. 79, 2727 (1983)

${ }^{34}$ T. Ichimura, Y. Mori, and K. Yoshihara, Chem. Phys. Lett. 104, 533 (1984); J. Chem. Phys. 83, 117 (1985); T. Ichimura, M. Takahashi, and Y. Mori, Chem. Phys. 114, 111 (1987). 
${ }^{35}$ M. Damm, H. Hippler, and J. Troe (preprint, 1987).

${ }^{36}$ L. Brouwer and J. Troe (preprint, 1987).

${ }^{37}$ K. M. Beck, A. Ringwelski, and R. J. Gordon, Chem. Phys. Lett. 121, 529 (1985).
${ }^{38}$ K. E. Shuler, G. H. Weiss, and K. Andersen, J. Math. Phys. 3, 550 (1962).

${ }^{39}$ H. O. Pritchard, J. Phys. Chem. 90, 4471 (1986).

${ }^{40} J$. E. Dove and J. Troe, Chem. Phys. 35, 1 (1978). 